\section{5 ans de I'Association} Alzheimer Suisse

Réunis à l'occasion des 25 ans de l'Association, les délégués ont adopté le rapport annuel. La stratégie nationale en matière de démence qui sera adoptée par le Dialogue de la Politique nationale de la santé à la fin de cet automne était au centre des activités politiques ces dernières années. En Suisse, les offres de prestations pour les personnes atteintes de démence et pour les proches soignants sont généralement bonnes mais insuffisantes. Il faudra aussi de nouvelles idées et initiatives. C'est ainsi que l'Association Alzheimer Suisse soutient un projet pilote visant à mettre en place des colocations pour des malades vivant seuls.

(L'Association Alzheimer Suisse)

\section{Pink RibbonCharity Walk 2013}

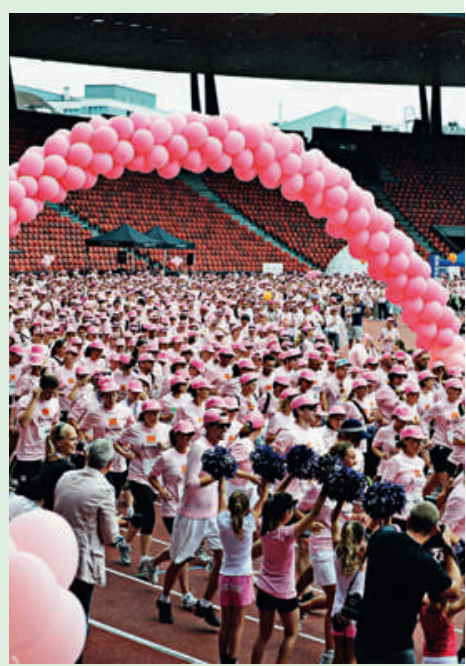

Am Sonntag, 29. September 2013, findet im Stadion Letzigrund Zürich der sechste «Pink RibbonCharity Walk» statt. Wie immer geht es beim Brustkrebs-Solidaritätslauf nicht ums Gewinnen, sondern um die Sensibilisierung der Früherkennung von Brustkrebs. Die Teilnehmenden möchten ihre Solidarität mit Brustkrebs-Patientinnen und deren Angehörigen kundtun und ein Zeichen setzen im Kampf gegen diese Krankheit. Weitere Informationen findet man auf www.pink-ribbon.ch

(Pink Ribbon Schweiz)

\section{Sexualität in der Pflege}

Menschen mit einer schweren Behinderung und der oft damit einhergehenden Pflegebedürftigkeit sind ebenso Menschen mit sexuellen Bedürfnissen. Diese Erkenntnis findet allmählich Eingang in die Gesellschaft - und ebenfalls in die Pflegetätigkeit. Denn die Pflege von schwerbehinderten Menschen stellt eine körperintensive, mitunter gar intime Angelegenheit dar. In der heutigen Pflegeausbildung werden sexuelle Bedürfnisse von pflegebedürftigen Menschen behandelt. Der Schweizer Berufsverband der Pflegefachfrauen und Pflegefachmänner hat zum Thema sexuelle Belästigung in der Pflege eine Broschüre herausgegeben: «Verstehen Sie keinen Spass, Schwester?». Darin lesen interessierte Pflegende unter anderem über ihre Möglichkeiten, mit derartigen Problemen umzugehen beziehungsweise sie erst gar nicht aufkommen zu lassen.

(My Handicap)

\section{Augengesundheit}

Die positive Beeinflussung der Augengesundheit durch Nutrazeutika wird seit vielen Jahren diskutiert. Viele Hinweise sprechen dafür, dass Karotinoide, die sich in der Netzhaut des Auges, der Macula lutea, anreichern, sowohl für eine optimale Funktion als auch für die Prävention der altersbedingten Makuladegeneration (AMD) eine wesentliche Rolle spielen. Eine aktuelle Studie (AREDS-2 Studie) ergab jedoch keine klaren positiven Effekte für die Supplementation. Dabei ist aber zu bedenken, dass die Probanden bereits an AMD erkrankt waren. Positiv ist jedoch, dass bereits geringste Mengen Lutein und Zeaxanthin, über die Nahrung eingenommen, das Risiko, an einer fortgeschrittenen AMD zu erkranken, um 26 Prozent verringerten. Das zeigt, dass eine Supplementa-

\section{Migration et assistance à domicile}

Pour pouvoir assurer une prise en charge à domicile de leurs proches âgés, de plus en plus de ménages suisses engagent du personnel soignant venant

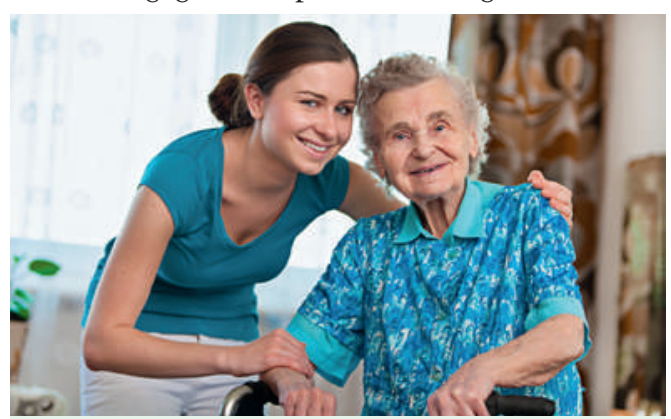

De plus en plus de ménages suisses engagent du personnel soignant venant d'Europe de l'Est.

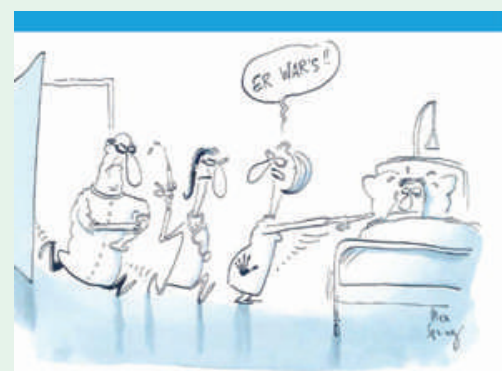

"Verstehen Sie keinen

Spass, Schwester?"

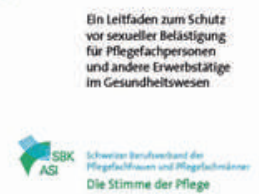

Eine neue Broschüre informiert Pflegende zum Thema «Sexuelle Belästigung in der Pflege».

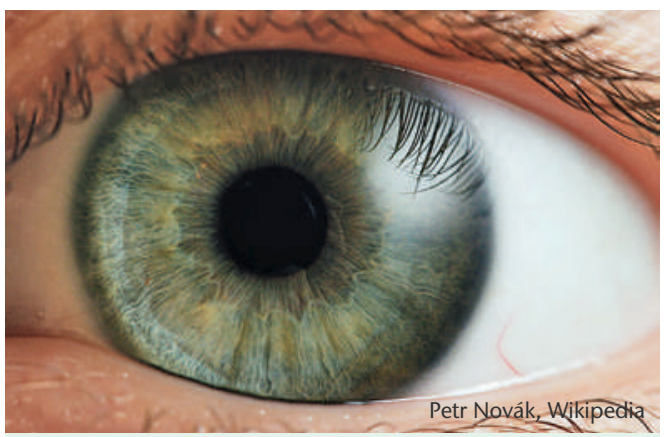

Bei einer Mangelsituation verringern Lutein und Zeaxanthin das Risiko einer fortgeschrittenen Makuladegeneration um $26 \%$.

tion besonders dort erfolgreich ist, wo eine Mangelsituation vorherrscht.

(European Nutraceutical Association)

d'Europe de l'Est, souvent à des conditions de travail précaires. Mais à quelles conditions les migrants ont-ils intérêt à prendre un emploi à l'étranger? Caritas préconise l'adoption de conditionscadres à différents niveaux pour ces emplois: ainsi, elle demande au Conseil fédéral et au Parlement de ratifier la convention internationale sur les conditions minimums de travail pour les travailleuses et travailleurs domestiques, qui permettra de reconnaître au personnel de maison un statut de salarié. Elle demande entre autres aussi d'instituer le respect de conditions de travail équitables, d'un salaire minimum et d'horaires réguliers de travail au personnel de soins migrant. 